\title{
Vehicle Sensor Self-Powered Technology Research Based on Piezoelectric
}

\author{
Sang Ying-Jun ${ }^{1}$, Wu Shangguang ${ }^{1}$, Li Man ${ }^{1}$, Gao Yang ${ }^{1}$, Li Haoxiang ${ }^{1}$, Huang Fei ${ }^{1}$ \\ Hao Yun-Rong ${ }^{2}$, Fan Yuan-Yuan ${ }^{2}$ \\ ${ }^{1}$ Faculty of Electronic and Electrical Engineering, Huaiyin Institute of Technology, Huaian 223003, China \\ ${ }^{2}$ Faculty of Mathematics and Physics, Huaiyin Institute of Technology, Huaian 223003, China \\ Corresponding author, e-mail: sangyingj@163.com
}

\begin{abstract}
As a result of the electric vehicles popularity and the development of vehicles intelligent, the number of vehicle sensors surge, meanwhile, many defects of traditional energy supply are increasingly prominent, such as pollution and maintenance difficulties. Taking into account the vehicle vibration exist everywhere, we use the piezoelectric technology to collect vibration energy, and designs a piezoelectric vibration energy harvesting system to be used to solve the energy problem of micro-power sensor. In this paper, the system structure and the theoretical model are analyzed, and the mathematical model of the system vibration frequency and the piezoelectric output have been put forward, then a piezoelectric energy harvesting device is designed on the basis of simulation analysis. Experiments have been done to test the performance of its power generation in the case of resonance. The results showed that the theoretical model proposed in this paper can be a good predictor of the output characteristics of the system. As the resonance frequency is $16.5 \mathrm{~Hz}$, acceleration is $0.5 \mathrm{~g}$, the maximum open circuit voltage of the system obtained is 3.5 volts, the optimum load resistance is $425 \mathrm{k}$, and the vibration energy collection device maximum load power is $14 \mathrm{uW}$. Conclusion: Greater energy could be caught to meet the vehicle sensor power supply needs with the use of super capacitor.
\end{abstract}

Keywords: environmental vibration, piezoelectric, cantilever, energy conversion, load power

Copyright $\odot 2015$ Institute of Advanced Engineering and Science. All rights reserved.

\section{Introduction}

With the development of the intelligent electric cars and auto sensor miniaturization, the number of sensors will dramatically increase and the sensors will spread around the car. Noding energy problem of a large number of micro sensors is becoming one of the problems to be solved in a hurry $[1,2]$. People hope that they can collect energy from the electric car's vibration environment and after transformation of sensors, the energy can supply power. Harvesting vibration energy is a popular way, which is currently studying technology, this way of power supply can prolong the life of low power consumption equipment and can not rely on batteries or wiring harness to separate the power supply equipment [3].

It is feasible to convert the energy of environmental vibration into electrical energy, and the vibration is everywhere, and the energy of the vehicle can be recovered from the vehicle. At present, there were reported on the use of different energy harvesting methods to improve the efficiency of the vibration energy collection in a lot of literature [4,5]. Because of the high availability, good performance and high output voltage [6], it is widely concerned about the new technology of using piezoelectric effect to deal with energy.

Piezoelectric vibration energy harvesting system can be used to solve the problem of micro power sensor [7, 8]. In this paper, a vibration energy harvesting device is designed, which is based on the combination of resonance and piezoelectric technology, and the power generation performance is tested. Experimental results show that, at the resonance frequency of $16.5 \mathrm{~Hz}$, acceleration is $0.5 \mathrm{~g}$, the maximum open circuit voltage of system is $3.5 \mathrm{~V}$, in the optimal load resistance to $425 \mathrm{k}$, the maximum load power of vibration energy collection device is $14 \mathrm{uW}$, system model well reflects the output characteristics of the system. 


\section{The System Structure and Mathematical Model}

\subsection{The System Structure and Principle}

The piezoelectric energy harvesting device based on vibration can be described by the structure shown in Figure 1(a). The device is composed of a piezoelectric cantilever beam with a mass block at the end. Mass blocks are used to excite and adjust the resonance frequency. The vibration of cantilever beam is caused by external vibration. The piezoelectric plate generates charge when it is subjected to an action of stress, which is integrated with the two part of the energy to increase the total energy of the output.

\subsection{Mathematical Model}

Piezoelectric cantilever beam structure is most sensitive to the ambient vibration, prone to forced vibration, when the vibration mechanics analysis, the vibration model caused by the cantilever beam under external excitation vibration can be described using spring - mass damping single degree of freedom system model [2] as shown in Figure 1(b). The model of vibration system is:

$$
\left.+2 \xi \omega_{\mathrm{n}} z(t)+\omega_{\mathrm{n}}^{2} z(t)=-\gamma\right)
$$

In the formula(1),

$y(t)=$ Based on the external environment vibration displacement

$z(t)=$ Relative vibration displacement of mass

$\xi=$ The damping ratio $\quad \xi=c / 2 \sqrt{\mathrm{km}} \quad c$ Represent damp

$\omega_{\mathrm{n}}=$ The angular frequency of the fundamental oscillation system $\omega_{\mathrm{n}}=\sqrt{K / m} \quad \mathrm{~K}$ is

effective elastic coefficient system $m=\Delta m+0.236 M^{[3]} \Delta m$ is the quality of the mass $M$ is the quality of the cantilever beam.

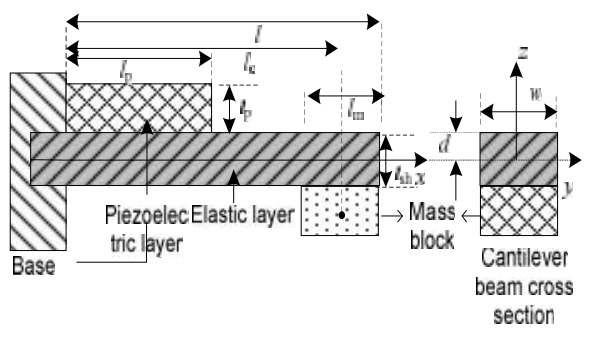

(a) The structure of the cantilever beam model

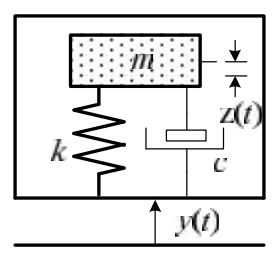

(b) Spring-Mass-Damping Model

Figure 1. Unequal model of single crystal CVPG

According to the cross section conversion method [4] and moment of inertia parallel axis theorem [5], using the equivalent section inertia moment I1, I2 equation of cantilever beam in the $x=0 \sim L_{P}$ and $x=L_{P} \sim L_{e}$ section, combined with structural mechanics theory [4], it is shows that the cantilever beam system effective elastic coefficient is:

$$
K=\frac{3 Y_{\mathrm{p}} I_{1} I_{2}}{\left(l_{\mathrm{e}}^{3}-\left(l_{\mathrm{e}}-l_{\mathrm{p}}\right)^{3}\right) I_{2}+\left(l_{\mathrm{e}}-l_{\mathrm{p}}\right)^{3} I_{1}}
$$
know.

Using the system fundamental frequency angular frequency of vibration formula we can

$$
\begin{aligned}
& \omega_{\mathrm{n}}=K_{1} \sqrt{\frac{K}{m}}=K_{1} \sqrt{\frac{3 Y_{\mathrm{p}} I_{1} I_{2}}{\left[\left(l_{\mathrm{e}}^{3}-\left(l_{\mathrm{e}}-l_{\mathrm{p}}\right)^{3}\right) I_{2}+\left(l_{\mathrm{e}}-l_{\mathrm{p}}\right)^{3} I_{1}\right](m+0.236 M)}} \\
& K_{1}=\text { Frequency correction factor. }
\end{aligned}
$$


The piezoelectric Equation [5] shows that when the cantilever beam is subjected to the inertial force of $Z$ direction, the piezoelectric layer will generate tension and compression in the $X$ direction, and the coupling electric field is formed in the $Z$ direction. For the piezoelectric strain $\delta=-d_{31} E_{3}$, it can be equivalent to a concentrated force at the end of a cantilever beam $F_{E}$, In formula (1), after adding an electric field force, we can get:

$$
m(t)+c z(t)+K z(t)=-m(t)-F_{\mathrm{E}}(t)
$$

According to the equation and relationship strain and bending moment, we can get $F_{\mathrm{E}}$

$$
=k_{1} U \quad k_{1}=4 \frac{d_{31} Y_{\mathrm{p}} I_{1}}{\left(2 l_{\mathrm{b}}-l_{\mathrm{p}}\right)\left(t_{\mathrm{p}}+2 d\right) t_{\mathrm{p}}} \text {. }
$$

The $F_{E}$ substituted into the formula (4) we can get:

$$
z=\frac{\omega^{2} y-k_{1} U / m}{-\omega^{2}+j 2 \xi \omega \omega_{\mathrm{n}}+\omega_{\mathrm{n}}^{2}}
$$

According to the principle of thermal equilibrium, the piezoelectric layer can be represented by the total energy of the system [6] produced by the infinitesimal unit, then using the relationship between cantilever beam by external forces and the relative displacement $\mathrm{z}[7$, 8], the relationship between the vibration acceleration and open circuit voltage of Piezoelectric vibration generator under the environmental vibration excitation is:

$$
\begin{aligned}
V_{\mathrm{o}} & =\left(\frac{K k_{2} \frac{K k_{2}}{C_{\mathrm{P}}} A_{i n}}{-\omega^{2}+\mathrm{j} 2 \xi \omega \omega_{\mathrm{n}}+\omega_{\mathrm{n}}{ }^{2}\left(1+k_{\mathrm{p}}\right)}\right) \\
A_{\text {in }} & =\text { Representing the external excitation of vibration acceleration, } A_{\mathrm{in}}=\omega^{2} y . \\
K_{2} & =\text { Voltage correction factor. }
\end{aligned}
$$

\section{Simulation Analysis}

Using MATLAB software to numerical simulation for Piezoelectric model the relationship between the piezoelectric cantilever resonance frequency, cantilever length, the width of the cantilever, the length of the mass, the height of the mass and structural parameters of piezoelectric ceramics is showed in the Figure 2.

From the Figure 2(a) it can be seen that resonance frequency is decreased with the increase of the length of the piezoelectric ceramic piece when the length of the cantilever beam quality piece is certain, resonance frequency is decreased with the increase of the length of the mass when the length of piezoelectric ceramics is under certain circumstances. From the Figure 2(b) it can be seen that the resonant frequency of a piezoelectric cantilever beam is increased with the increase of piezoelectric ceramic chip thickness and the thickness of the metal gasket.

From the Figure 2(c), it can be seen that when the height of the small mass is same, the resonance frequency of the piezoelectric cantilever beam increases with the increase of the width of the cantilever beam; while the width of the cantilever beam is determined, the resonance frequency of piezoelectric cantilever beam does not change as the change of the height of the small mass. 


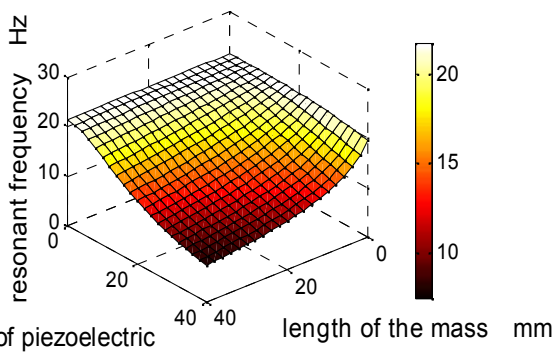

length of piezoelectric ceramic piece $\mathrm{mm}$

(a) The relationship between resonant frequency - length of piezoelectric ceramic piece- length of the mass

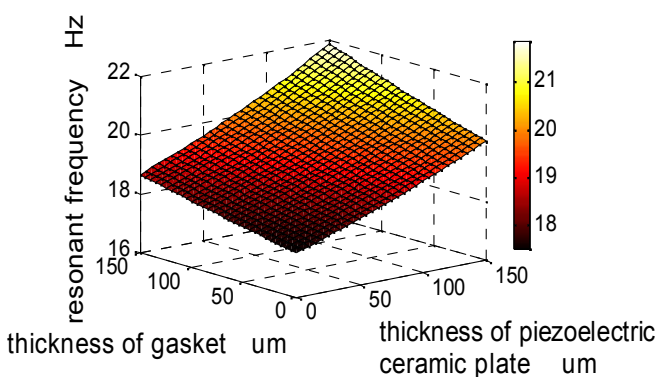

(b) The relationship between resonant frequency - thickness of piezoelectric ceramic plate - thickness of gasket

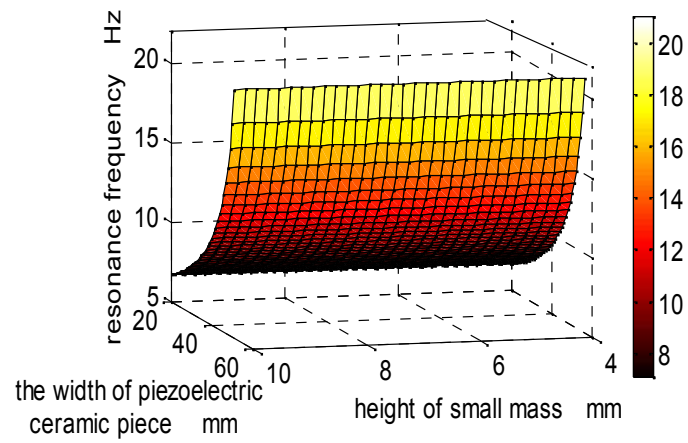

(c) The relationship between resonance frequency- the width of piezoelectric ceramic piece height of small mass

Figure 2. Resonance frequency and cantilever beam structure parameters

\section{Test and analysis of system performance}

\subsection{Design and Experiment}

The simulation analysis shows the resonance frequency of the device is associated with the structure parameters of the cantilever arm. By changing the parameters of the piezoelectric sheet and the substrate sheet, such as length, width, thickness etc, the resonant frequency of the system can be adjusted. The System resonant frequency is designed $12 \mathrm{~Hz}$. Combining with the simulation analysis; we determine the size parameters of the piezoelectric cantilever beam as the Table 1 showing:

Table 1. The parameters of the piezoelectric energy harvesting devices

\begin{tabular}{ccccc}
\hline The parameter name & value & The parameter name & value \\
\hline The length of the cantilever beam $(\mathrm{mm})$ & 55 & The width of the cantilever beam $(\mathrm{mm})$ & 30 \\
The length of the piezoelectric element $(\mathrm{mm})$ & 40 & The width of the piezoelectric element $(\mathrm{mm})$ & 30 \\
The thickness of the piezoelectric layer $(\mathrm{mm})$ & 0.1 & The weight of the mass $(\mathrm{g})$ & 18 \\
The thickness of the substrate $(\mathrm{mm})$ & 0.15 & The height of the mass $\mathrm{mm}$ & 15 \\
\hline
\end{tabular}

In order to collect and analyze data easily, we use the virtual signal generator to generate a sine wave. We will program to realize the adjustment of the sine wave frequency and the amplitude. Through the digital to analog converter (D/A) excitation source we can simulate external vibration source. The piezoelectric cantilever beam structure which output voltage vibrating with the exciter. We collect the data drawing and save it through the data acquisition card to analyze the data, so we can analyze the relationship among the system output voltage, vibration amplitude and vibration frequencies. The structure diagram of experimental system is presented in the Figure 3(a), and the experimental picture is shown in the Figure 3(b), the oscilloscope displays a waveform of the piezoelectric open circuit output voltage. The energy 
conversion circuit is consist of DF005S Rectifier Bridge, 0.047F large capacitances which are used to store electrical energy and voltage regulator circuit constituted with TPS62200.

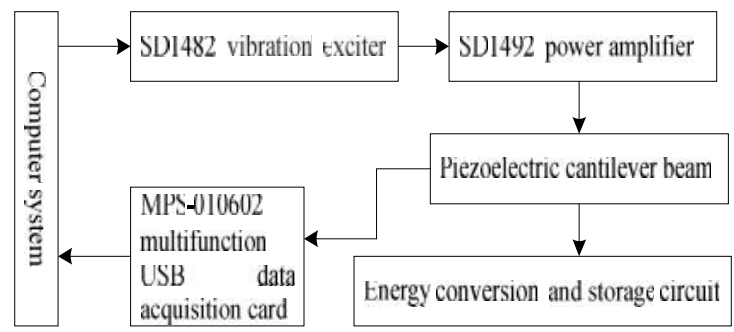

(a)
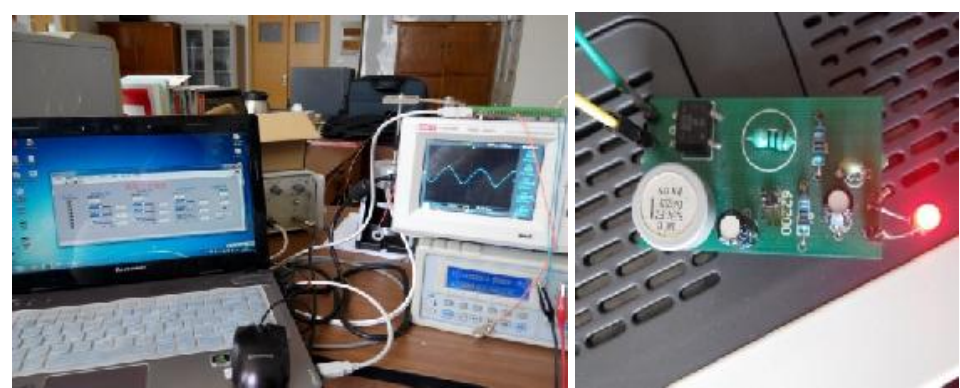

(b)

Figure 3. (a) The structure diagram of experimental system; (b) The experimental picture

\section{The Analysis of Experimental Results}

The acquisition of the systems theory simulation value doesn't consider the case of a correction coefficient. If $\mathrm{K} 1=\mathrm{K} 2=1$, the theoretical value of the resonance frequency is $16 \mathrm{~Hz}$, the resonant frequency measured by the experiment is $16.5 \mathrm{~Hz}$. When the vibration acceleration is $0.5 \mathrm{~g}$, the peak voltage of the piezoelectric cantilever working in the resonance frequency is about $3.5 \mathrm{~V}$, which is lower than the theoretical value of $4 \mathrm{~V}$ slightly. The relation curve of the output peak voltage and the vibration frequency is shown in Figure 4. We can see when the vibration frequency within $(10-24 \mathrm{~Hz})$ of the piezoelectric cantilever beam power generation system, in other words, when it close to the resonance frequency, can we get higher output voltage whose peak voltage is more than $2,5 \mathrm{v}$. However, no matter the frequency is too large or too small, output voltage is small.

The numerical simulation by Matlab and PSPICE circuit simulation have given the system's optimal load about $500 \mathrm{~K}$ in the resonant frequency, while $425 \mathrm{~K}$ is the experimentally measured and the instant power is $14 \mathrm{uW}$. system load voltage and load resistance shown in Figure 4. As the figure shown, the value of the load voltage increases as the load increases, while load power is rising in the first and at the optimal load resistance is about $425 \mathrm{~K}$ reach the peak. The load power will decrease when the load resistance is greater than $425 \mathrm{~K}$.

It is shown in the experiments that the experimental value is higher than the value obtained by our theory. The reason is, because the cantilever fixed base increased to some extent, start-up portion of the cantilever length, so that the resonance frequency of the theoretical value obtained is slightly lower than the actual, while the output voltage will drop slightly is the main reason, in addition to the calculation error and experimental reading error. Consider these factors, based on the experimental results analysis, the system model proposed amendment, take $\mathrm{K} 1=0.97, \mathrm{~K} 2=0.89$, the model can be modified to better reflect the system's output characteristics. 


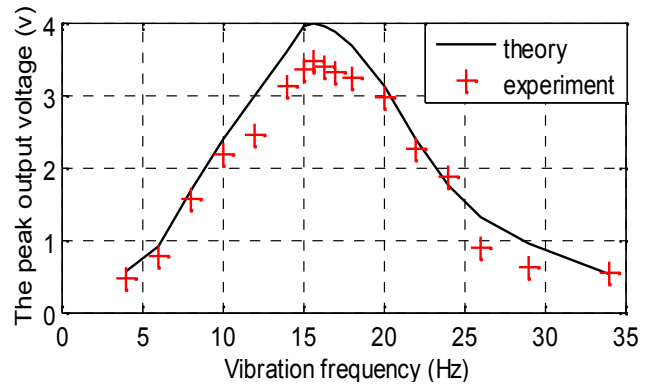

(a) The relation curve about the peak output voltage and frequency of vibration

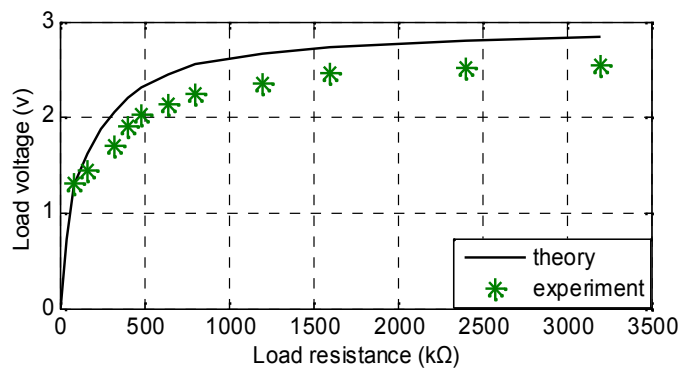

(b) The relation curve about system load voltage and load resistance

Figure 4. The system load voltage and load resistance

\section{Conclusion}

This article aims at the power needs of automotive micro-sensors, piezoelectric technology is proposed recovery vehicle vibrational energy in the environment. This article studies the mathematical model based on piezoelectric energy harvesting systems, and structural parameters of the system and its relationship resonance simulation analysis, design a combination of resonance and vibration energy collecting device piezo technology, the resonance frequency $16.5 \mathrm{~Hz}$, acceleration of $0.5 \mathrm{~g}$, the maximum open circuit voltage of $3.5 \mathrm{~V}$ is obtained when the optimum load resistance is about $425 \mathrm{k}$, vibration energy harvesting load power is about $14 \mathrm{uW}$, the results not only validate the theoretical model accuracy but also indicating that the system can meet the energy more powerful batch mode of operation of supply and demand on-board sensors.

\section{Acknowledgments}

This work was supported in part by National Natural Science Foundation of China (Grant No.51307070).

\section{References}

[1] Williams CB, Yates RB. Analysis of a micro electric generator for micro systems. Sensors and Actuators: Physical. 1996; 52(1-3): 8-11.

[2] Hibbeler RC. Mechanics of materials. USA: Pearson Prentice Hall publisher. 2005.

[3] Yu Hua, Zhou Jielin, Deng Licheng, Wen Zhiyu. A vibration-based MEMS piezoelectric energy harvester and power conditioning circuit. Sensors. Switzerland. 2014; 19(14): 3323-3341.

[4] Junwu Kan, Ke Hong Tang, Shu-yun WANG, et al. The piezoelectric cantilever beam generating device modeling and simulation analysis. Optics and Precision Engineering. 2008; 16(1): 71-75.

[5] Xing Jiang Zhao, Zhiyu Wen. Adjustment based on vibration energy collector resonant frequency of the piezoelectric material. Piezoelectric and sound and light. 2013; 35(2): 241-244.

[6] Jiang Bo Yuan, Xiao Bo Shan, Tao Xie. A single crystal piezoelectric cantilever generators experiments. Optics and Precision Engineering. 2009; 17(5): 1072-1077.

[7] Li Qing Fang, Lei Zhang. Single crystal piezoelectric cantilever equivalent viscous damping modeling and experiments. Optics and Precision Engineering. 2014; 22(3): 641-648.

[8] Guang Ming Cheng, Xinhui Li, Haibin Zhang, Ping Zeng, Jian Ming Wen. The effect of cantilevered bimorph vibrator clamping length change dynamic characteristics. Optics and Precision Engineering. 2014; 22(5): 1296-1303. 